\title{
Patients' perceptions of maternal healthcare acceptability: A scoping review protocol
}

Joy Blaise BUCYIBARUTA, MMed (Paed), FCPaed (SA), MPH, MBChB. ${ }^{1}$ Leah MAIDMENT, MBChB student. ${ }^{1}$ Carl August Daniel HEESE, Bsc student. ${ }^{1}$ Mmapheko Doriccah PEU, PhD, MCur, BCur Hons, FANSA. ${ }^{1}$ Lesley BAMFORD, DrPH, FCPaed (SA), BSocSci (Hons), MBChB. ${ }^{1,2}$ Annatjie Elizabeth van der WATH, PhD, MCur, BCur. ${ }^{1}$ Estelle GROBER, BLISc. ${ }^{1}$

${ }^{1}$ University of Pretoria, Faculty of Health Sciences.

${ }^{2}$ National Department of Health, South Africa.

Note. This is a preprint that has yet to complete the peer review process.

Submitted to Meta-Psychology. Participate in open peer review by commenting through hypothesis directly on this preprint. The full editorial process of all articles under review at Meta-Psychology can be found following this link:

https://tinyurl.com/mp-submissions

You will find this preprint by searching for the first authors' name.

Corresponding author: Joy Blaise Bucyibaruta, u19375370@ tuks.co.za and +27739160808. 


\begin{abstract}
Healthcare acceptability is an important concept in health sciences including psychology, yet, it remains controversial and poorly understood by all health researchers. Healthcare acceptability cuts across all health disciplines and refers to human behavioural constructs such as attitude, trust and respect, which are expressed when patients and health professionals interact. Many studies have described the acceptability of maternal healthcare, but there is no universally accepted definition. We describe a protocol for a scoping review of existing literature to shed-light-on the definition and conceptualisation of maternal healthcare acceptability from patients' perspectives. We will employ a search strategy, with eligibility criteria, to search for relevant articles from electronic and grey literature. Two researchers will independently screen the retrieved articles using Rayyan software and chart data from included articles. An inter-reviewer agreement of $80 \%$ will be deemed appropriate. We will interpret key findings in line with available evidence, while being consistent with the research purpose. We will discuss the study's limitations, implications for practice and propose future research projects.
\end{abstract}

\title{
Keywords
}

Acceptability, attitudes, expectations, experiences, healthcare provider interactions, healthcare systems and policy interactions, maternal healthcare, perceptions, support 


\section{Main text}

This is an Open Access article distributed in accordance with the Creative Commons Attribution Non Commercial (CC BY-NC 4.0) license, which permits others to distribute, remix, adapt, build upon this work non-commercially, and license their derivative works on different terms, provided the original work is properly cited and the use is non-commercial. For more detail on this subject, see: http://creativecommons.org/licenses/by-nc/4.0/

\section{Introduction}

The concept of 'acceptability of healthcare' is becoming increasingly relevant in health sciences, including psychology, to improve healthcare service delivery to the population (Cameron et al., 2017; Sekhon, Cartwright, \& Francis, 2017; Shaw, Larkin, \& Flowers, 2014). The concept of acceptability of healthcare cuts across all countries and all healthcare disciplines and is undeniably important when planning, implementing and monitoring healthcare interventions (Cameron et al., 2017; Shaw et al., 2014).

Acceptability of healthcare reflects the quality of interactions between the patient and the health provider the community or the health systems (Gilson, 2007). Those interactions can be described using terms conveying beliefs, such as respect, privacy, confidentiality, trust, understanding and support (Dyer, Owens, \& Robinson, 2016; Murphy \& Gardner, 2019). Furthermore, the interpretation of those interactions is often subjective and depend on the perceived quality of received or anticipated healthcare (Dyer et al., 2016; Murphy \& Gardner, 2019). Many of the terms used to describe beliefs have overextended meanings and some researchers have proposed categorising these terms under specific constructs of acceptability by applying best-fit theory (Gilson, 2007; McIntyre, Thiede, \& Birch, 2009). The nature of patient-provider interactions is multifaceted (Bucyibaruta et al., 2018), which complicates the concept of 'acceptability of healthcare'. The complicated nature of the 
concept has resulted in there being no universally accepted application within the wider community of health professionals. More research is needed to create a uniform understanding of healthcare acceptability that can be practically applied.

The concept of healthcare acceptability — also referred to as cultural access — was introduced in the early 1980's as one of the three dimensions of access to healthcare (Penchansky \& Thomas, 1981). The other two dimensions are affordability or financial access, and availability or physical access (Bucyibaruta et al., 2018; McIntyre et al., 2009; Silal, Penn-Kekana, Harris, Birch, \& McIntyre, 2012). Acceptability was originally described as "the best fit fulfilment of healthcare expectations between the patient and the healthcare system" (Penchansky \& Thomas, 1981). Following this initial description, many attempts were made to refine the definition of healthcare acceptibility (Dillip et al., 2012; Donabedian, 2002; Kozarewicz, 2014; Kyei-Nimakoh, Carolan-Olah, \& McCann, 2017; Rothstein et al., 2016; Russell et al., 2013; Sekhon, Cartwright, \& Francis, 2018; Staniszewska et al., 2010). Despite these efforts, there is still no consensual definition of healthcare acceptibility within the broader community of health researchers.

The lack of a clear definition of healthcare acceptability impedes how the concept is applied at the level of definite healthcare such as maternal healthcare. While different types of healthcare services share common characteristics, maternal healthcare has certain unique aspects as far as acceptability is concerned. For example, maternal healthcare comprises antenatal care, delivery and post-natal healthcare services. Acceptability of maternal healthcare thus needs to match specific expectations and experiences of mothers attending antenatal, delivery and post-natal healthcare services. Although much research has been done on the acceptability of maternal healthcare (Al-Mujtaba et al., 2020; Balde et al., 2017; Cummins et al., 2021; Feinberg, Smith, \& Naik, 2009; Grant et al., 2017; Påfs et al., 2015; 
Sripad, Warren, Hindin, \& Karra, 2019), each study has used a different definition of maternal healthcare acceptability.

Evidently, researchers still need to agree on a widely accepted definition of maternal healthcare acceptability. Women often go through psychological distress resulting from various stressors and demands that are difficult to cope with during pregnancy, delivery and immediately post-partum (Staneva, Bogossian, \& Wittkowski, 2015; Traylor, Johnson, Kimmel, \& Manuck, 2020). With these multiple stressors in mind, acceptable maternal healthcare is difficult to define, especially when most attention is given to high-risk or complicated pregnancies or deliveries (Alderdice, McNeill, \& Lynn, 2013; Hadfield \& Wittkowski, 2017). Defining acceptability of maternal healthcare is important for health researchers including psychology researchers to advance and support appropriate health practice in maternal healthcare (Sekhon et al., 2018).

This protocol outlines the proposed strategy for reviewing existing literature to shedlight-on how maternal healthcare acceptability is defined and conceptualised. The specific objectives will include:

1. Identify gaps in defining the concept of maternal healthcare acceptability.

2. Explore the contextual understanding of maternal healthcare acceptability.

3. Ascertain the implications of acceptability for maternal healthcare practice.

\section{Methods}

This study is embedded in a larger $\mathrm{PhD}$ research project applying mixed methods, including a scoping review, and has been approved by the Faculty of Health Sciences Research Ethics Committee, University of Pretoria (No: 545/2019). This protocol is submitted for registered report and will be conducted once in-principle acceptance (IPA) is provided by Meta-Psychology. The protocol is also registered on Open Science Framework 
(OSF) to increase research transparency and to avoid unintended duplication of reviews. This study will be conducted in line with the registered report's guidelines and will be subject to ethical and policy considerations of Meta-Psychology.

\section{Study design}

Scoping review is an appropriate method to organize and summarize existing literature in an orderly and replicable way to identify gaps in the literature and to answer a broader research question (Armstrong, Hall, Doyle, \& Waters, 2011; Dijkers, 2015). This scoping review will be conducted in six steps as described by Arksey and O'Malley (2005). These steps include: (i) identifying the research question, (ii) identifying relevant studies, (iii) selecting eligible studies, (iv) charting the data, (v) collating and summarizing the results, and (vi) consulting with experts in the field [optional]. We plan to engage with experts to improve the usefulness of our findings for future interventions. This study will also be guided by the scoping review framework developed by the Joanna Briggs Institute to enhance methodological quality (Tricco et al., 2018).

\section{Identifying the research question}

To establish the current understanding of maternal healthcare acceptability, this scoping review will answer the following questions:

1. How is maternal healthcare acceptability defined and conceptualized?

2. What are contextual understandings of maternal healthcare acceptability?

3. What are implications of acceptability for maternal healthcare practice?

\section{Identify relevant studies}

To ensure a comprehensive search, the principal investigator (PI) and two co-authors will independently search for relevant articles using online databases including 
MEDLINE/PubMed, Cochrane Library, Google Scholar, CINAHL, etc. The researchers will apply a snowball strategy by checking the reference lists of retrieved studies as well as 'cited by' articles to identify additional studies. Researchers will search relevant grey literature including dissertations/theses (ProQuest Dissertations \& Theses Global), conference abstracts (EMBASE Conference Abstracts, Conference Proceedings), PowerPoint presentations, magazines, websites such as the WHO, departments of health in different countries, Google websites and unpublished work on the topic.

Relevant studies will be identified according to eligibility criteria and the search strategy developed by the PI. The PI will ensure that the other two researchers understand the eligibility criteria and search strategy before searching for literature and identifying studies themselves. Relevant studies will be identified iteratively. After retrieving about 1000 articles, the researchers will focus on the other steps of the scoping review. Additional relevant studies will be identified based on preliminary findings, or if the researchers and experts agree that further search is needed.

\section{Selection of eligible studies}

We will adopt an open strategy when searching literature on acceptability of maternal healthcare, but will only include sources written in English because the researchers who will be screening articles are literate in English. Inclusion of articles will not be limited by study design or year of publication. Similarly, we will include articles without appraising methodological quality or assessing risk bias, in line with scoping review methodology (Armstrong et al., 2011). 


\section{Eligibility criteria}

We will screen identified studies using carefully developed eligibility criteria to ensure that relevant studies are included. Eligibility criteria have been determined using Population-Concept-Context (P-C-C) criteria as described in table 1.

Table 1: Eligibility criteria for including studies on acceptability of maternal healthcare.

\begin{tabular}{l|l}
\hline CRITERIA & DESCRIPTION \\
\hline Population & Women aged 18 years and above seeking maternal healthcare \\
Concept & $\begin{array}{l}\text { Acceptability of maternal healthcare (antenatal; delivery; post-partum) } \\
\text { Context }\end{array}$ \\
\hline
\end{tabular}

\section{Exclusion criteria}

Articles will be excluded based on the following criteria:

- Studies reporting on female population aged younger than 18 years including adolescent or teenage pregnancies.

- Studies reporting on acceptability of services other than maternal healthcare or maternal healthcare acceptability beyond antenatal, delivery and immediate (within 42 days) post-partum.

- Studies without full-text.

Table 2: Search strategy used to search online databases for articles related to acceptability of maternal healthcare. 


\begin{tabular}{|c|c|c|}
\hline Eligibility criteria & Description & \\
\hline \multirow[b]{2}{*}{ Population } & $\begin{array}{l}\text { Keywords or MeSH } \\
\text { terms }\end{array}$ & "Women" \\
\hline & $\begin{array}{l}\text { Synonymous or proxy } \\
\text { words }\end{array}$ & $\begin{array}{l}\text { "Mothers", "females", "women of } \\
\text { reproductive age", etc. }\end{array}$ \\
\hline \multirow{3}{*}{ Concept } & $\begin{array}{l}\text { Keywords or MeSH } \\
\text { terms }\end{array}$ & "Acceptability of maternal healthcare" \\
\hline & $\begin{array}{l}\text { Synonymous or proxy } \\
\text { words for acceptability }\end{array}$ & $\begin{array}{l}\text { "Acceptable / unacceptable", "respectful } \\
\text { / disrespectful", "trust / distrust", } \\
\text { "supportive / unsupportive", "caring } \\
\text { /uncaring", "perception/experience", etc. }\end{array}$ \\
\hline & $\begin{array}{l}\text { Synonymous or proxy } \\
\text { words for maternal } \\
\text { healthcare }\end{array}$ & $\begin{array}{l}\text { "Pregnancy", "labour", "delivery", } \\
\text { "post-partum", "maternal healthcare } \\
\text { services" "antenatal care", "PMTCT", } \\
\text { "mental health in pregnancy", } \\
\text { "breastfeeding", etc. }\end{array}$ \\
\hline \multirow{4}{*}{ Context } & $\begin{array}{l}\text { Keywords or MeSH } \\
\text { terms }\end{array}$ & $\begin{array}{l}\text { Specific country. Example: "South } \\
\text { Africa", Zimbabwe", "Malawi", } \\
\text { "Rwanda", "United States of America", } \\
\text { "Canada", "United Kingdom", etc. }\end{array}$ \\
\hline & $\begin{array}{l}\text { Synonymous or proxy } \\
\text { words }\end{array}$ & $\begin{array}{l}\text { Province, town or healthcare facility in a } \\
\text { specific country. Examples: "Gauteng", } \\
\text { "Western Cape", "KwaZulu-Natal", } \\
\text { "Mpumalanga", "Johannesburg", "Cape } \\
\text { Town", "Durban", "Secunda" "Chris } \\
\text { Hani Baragwanath", "Steve Biko", etc. }\end{array}$ \\
\hline & $\begin{array}{l}\text { Keywords or MeSH } \\
\text { terms }\end{array}$ & $\begin{array}{l}\text { Worldwide or specific continent: } \\
\text { "global", Africa", Europe", etc. }\end{array}$ \\
\hline & $\begin{array}{l}\text { Synonymous or proxy } \\
\text { words for maternal } \\
\text { healthcare }\end{array}$ & $\begin{array}{l}\text { Sub-regions within a continent. } \\
\text { "SADEC", "Sub-Saharan Africa", } \\
\text { "North Africa", "Western Europe", } \\
\text { "North America", etc. }\end{array}$ \\
\hline Boolean operators & “OR”, “AND”, "NOT” & \\
\hline
\end{tabular}

\section{Search strategy}

To ensure that all relevant studies were identified, we developed a search strategy using specific keywords or Medical Subject Headings (MeSH) terms in various combinations (Table 2). The PI and two independent researchers will conduct a pilot search, testing if the search strategy works well on different online databases. The researchers may refine the search strategy by using synonymous and/or proxy words to optimize the retrieval of relevant articles. 


\section{Level one screening}

After three researchers have independently identified relevant studies, all the identified studies will be merged into a single Endnote library. The PI will remove duplicates and import the merged Endnote library into Rayyan software for level one screening. Two researchers will independently screen the titles and abstracts of identified articles. Initially, 100 articles will be screened and an agreement level of $80 \%$ will be deemed acceptable. Once the reviewers agree on the screening process, the rest of the articles will be screened. The screening will be blinded and the PI will resolve any conflicts between the two independent researchers.

\section{Level two screening}

Once the titles and abstracts have been screened, the PI will export the included articles from Rayyan to Endnote, where the full-text PDFs will imported for each included article. The full-texts will be exported to Rayyan software for level two screening to identify articles that meet the inclusion or exclusion criteria. The screening process will be blinded with an agreement level of $80 \%$ between the two independent researchers deemed to be appropriate. The PI will resolve any conflicts occurring between the two screeners during the full-text screening.

\section{Searching database}

The PI developed a search database to document search histories (Table 3). 
Table 3: Searching database to document the search histories used to identify articles on acceptability of maternal healthcare.

\begin{tabular}{l|l|l|l|l}
\hline $\begin{array}{l}\text { Search } \\
\text { ID\# }\end{array}$ & Dates & $\begin{array}{l}\text { Number of studies } \\
\text { retrieved } \\
\text { (excluding } \\
\text { duplicates) }\end{array}$ & $\begin{array}{l}\text { Number of studies } \\
\text { selected after } \\
\text { screening level one }\end{array}$ & $\begin{array}{l}\text { Number of studies } \\
\text { included after } \\
\text { screening level two }\end{array}$ \\
\hline S\#1 & & & & \\
S\#2 & & & & \\
S\#3 & & & & \\
S\#4 & & & & \\
S\#5 & & & \\
Etc. & & & \\
\hline
\end{tabular}

\section{Charting the data}

For each identified study, data will be extracted to provide a logical and descriptive summary of relevant information. Key information will be extracted from the articles using a data charting form (Table 4). The PI will create a google document with all the data headings from the charting form. He will then invite two researchers to complete it independently. The PI and two researchers will conduct a pilot of the data charting process. An agreement level of $80 \%$ between the researchers will be considered appropriate before continuing with charting the rest of the articles. Any conflict between researchers will be resolved by the PI. The two researchers will submit their answers and the PI will review the answers in a google sheet, which will then be exported into Stata software for descriptive analysis.

Table 4: Charting form used to extract data from retrieved articles on acceptability of maternal healthcare.

\begin{tabular}{ll}
\hline Data heading & Description \\
\hline Title of study & Title of the article or study \\
Author/s & Name of author/s \\
Publication year & Year that the article was published \\
Study design & $\bullet$ Qualitative \\
& - Quantitative
\end{tabular}




\begin{tabular}{|c|c|}
\hline Data heading & Description \\
\hline & $\begin{array}{l}\text { - Mixed methods } \\
\text { - Scoping review } \\
\text { - Systematic review } \\
\text { - Meta-analysis } \\
\text { - Unknown }\end{array}$ \\
\hline Publication type & $\begin{array}{l}\text { - Journal } \\
\text { - } \text { Book } \\
\text { - Website } \\
\text { - Conference proceedings } \\
\text { - Unpublished } \\
\text { - Other (specify) }\end{array}$ \\
\hline Keywords & Keywords used by author/s \\
\hline Context & Study setting or country \\
\hline Type of maternal healthcare & $\begin{array}{l}\text { Antenatal (specify) } \\
\text { Labour \& delivery (specify) } \\
\text { Post-natal (specify) }\end{array}$ \\
\hline $\begin{array}{l}\text { Definition of maternal healthcare } \\
\text { acceptability }\end{array}$ & $\begin{array}{l}\text { Author/s provide/s the definition of maternal } \\
\text { healthcare acceptability (Yes or No) }\end{array}$ \\
\hline $\begin{array}{l}\text { Application of maternal healthcare } \\
\text { acceptability definition }\end{array}$ & $\begin{array}{l}\text { Author/s apply/s the definition of healthcare } \\
\text { acceptability in general (Yes or No) }\end{array}$ \\
\hline $\begin{array}{l}\text { Type of interactions with the } \\
\text { mothers }\end{array}$ & $\begin{array}{l}\text { - Mothers-community interactions } \\
\text { - Mothers-health provider interactions } \\
\text { - Mothers-health systems/policy interactions }\end{array}$ \\
\hline $\begin{array}{l}\text { Components of mothers- } \\
\text { community interactions }\end{array}$ & $\begin{array}{l}\text { - Support from husband or partner (yes or no) } \\
\text { - Support from family (yes or no) } \\
\text { - Support from community (yes or no) } \\
\text { - Other (specify) }\end{array}$ \\
\hline $\begin{array}{l}\text { Components of mothers- health } \\
\text { provider interactions }\end{array}$ & $\begin{array}{l}\text { - Language barrier } \\
\text { - Respecting privacy } \\
\text { - } \text { Assistance in labour } \\
\text { - } \text { - Bulking to health worker in private } \\
\text { - Being shouted at } \\
\text { - Being hit, slapped or pinched } \\
\text { - Health worker not respecting other patients } \\
\text { - Health worker not respecting me } \\
\text { - Other (specify). }\end{array}$ \\
\hline $\begin{array}{l}\text { Components of mothers- health } \\
\text { systems and policy interactions }\end{array}$ & $\begin{array}{l}\text { - } \quad \text { Dirty facilities } \\
\text { - } \text { Satisfied with received services } \\
\text { - } \text { Allowed to have companion during labour } \\
\text { - } \quad \text { Referred for follow up care } \\
\text { - } \text { Informed about child-care grant } \\
\text { - Other (specify) }\end{array}$ \\
\hline Practical implications & $\begin{array}{l}\text { - Yes (if yes; specify) } \\
\text { - No }\end{array}$ \\
\hline \multicolumn{2}{|l|}{ Comments } \\
\hline Conclusion & - Maternal healthcare acceptability \\
\hline
\end{tabular}




\section{Collating, summarizing and reporting the results}

The scoping review will be conducted in line with the Preferred Reporting Items for Systematic reviews and Meta-Analyses extension for Scoping Reviews (PRISMA-ScR)

(Liberati et al., 2009). The researchers will use the PRISMA-ScR flow diagram to demonstrate the process from the identification, inclusion and retention of articles fulfilling all eligibility criteria (Figure 1).

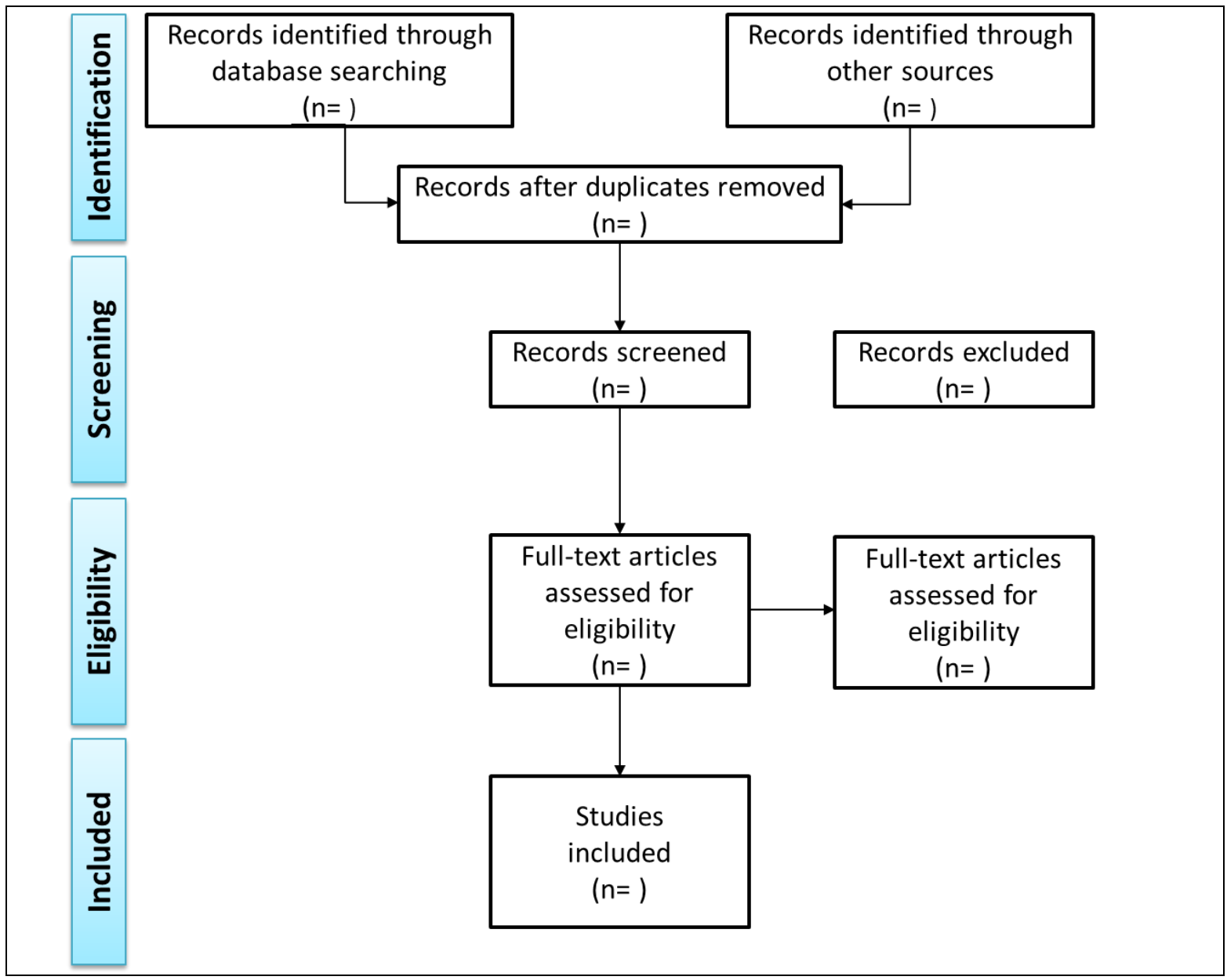

Figure 1: PRISMA-ScR flow diagram

The results will be presented in either a graphical/charted or tabular form. In addition, the researcher will provide a narrative summary accompanying the tabulated and/or charted 
results to highlight how the results are linked to the objectives and research questions of this study.

\section{Consultation exercise with experts}

A consultation exercise has been planned to engage with experts in the field through emails, one-to-on virtual meetings or through the Open Science Framework (OSF). Using consultations, we will regularly record thoughts, opinions and experiences from experts on maternal healthcare acceptability. These consultations should enhance the findings of the scoping review and identify additional references that may be included in this study. We will also ask experts their opinions on future research projects, policy decision-making and strengthening of health system practices.

\section{Ethics and dissemination}

This study will be conducted under an approved ethics certificate. The results will be presented at relevant conferences and published in a peer-reviewed journal.

\section{Discussion}

This study will identify gaps in the literature on acceptability of maternal healthcare, focusing on conceptual understanding and implications for practice of maternal healthcare acceptability in the context of South Africa and globally. A scoping review is an appropriate method to answer the broad aims of this research (Armstrong et al., 2011). The review will describe current definitions of maternal healthcare acceptability. The main results and evidence will be summarized in line with eligibility criteria (population-concept-context) (Dijkers, 2015). Findings will be discussed in relation to key stakeholders, including patients, communities, providers and health managers or policy makers. We will consult with experts 
to consider practical solutions for improving maternal healthcare acceptability (Arksey \& O'Malley, 2005).

\section{Strengths and limitations}

\section{Strengths}

Scoping reviews are a suitable evidence synthesis method to answer broad research questions. A thoughtful and rigorous protocol with clear stages will guide implementation of this project to reach the study objectives. To avoid bias, we have defined clear eligibility criteria, a detailed search strategy and composed a comprehensive data charting form. These steps also promote transparency and reproducibility. From these findings, we will be able to suggest future research studies such as systematic reviews or meta-analysis to address specific aspects of maternal healthcare acceptability.

\section{Limitations}

This study is conditional on Ethics Approval Reference No: No: 545/2019 for a PhD research project excluding young pregnant women aged younger than 18 years old. We will exclude studies on acceptability related to pregnancy, delivery and post-partum in teenagers. This will result in exclusion of critical information on acceptability of healthcare acceptability in pregnant adolescents. Another limitation is to omit studies on acceptability of maternal healthcare published in languages other than English. This may result in elimination of important studies on this topic.

\section{Data Availability}

To ensure transparency and reproducibility, all data generated or analyzed during this study will be included in the published scoping review article. This will include a list of 
included and excluded articles with reasons for excluding studies. We will also be documenting the search history and keep an excel spreadsheet of the charted data.

\section{Author Contributions}

Dr Joy Blaise Bucyibaruta is the PI and corresponding author. He is the project administrator and he was involved in conceptualization, data curation, formal analysis, investigation, methodology, writing — original draft preparation, and subsequent corrections —, editing, formatting and approval of article submission.

Ms Leah Maidment and Mr Carl Heese are the co-authors of this study. They have been recruited to get involved in the literature search, level one and two screening, data charting, proof-reading and approval of article submission.

Prof Doriccah Peu Prof Lesley Bamford read an article as a supervisor and cosupervisor respectively. They also provided some suggestions on how to improve the article.

Prof Annatjie van der Wath read an article as an external researcher and provided some suggestions on how to improve the article

Ms Estelle Grober is an information specialist and she has been recruited to get be involved in the literature search. She will also assist the PI to resolve the conflicts in screening and data charting between the two researchers to ensure the search reliability.

\section{Acknowledgments}

We would like to acknowledge the University of Pretoria, Faculty of Health Sciences for ethical approval and other supports to effectively conduct this study. Special thanks to the Tuks Undergraduate Research Forum (TURF) for recruiting independent researchers and to 
the Office of Deputy Dean: Research and Postgraduate Studies for organising training on evidence synthesis and use of Rayyan software. Dr. Cheryl Tosh for editing and formatting.

\section{Conflict of Interest Statement}

Authors declare no conflict of interest.

\section{Funding or sources of support}

No grant received for this article 


\section{References}

Al-Mujtaba, M., Shobo, O., Oyebola, B. C., Ohemu, B. O., Omale, I., Shuaibu, A., \& Anyanti, J. (2020). Assessing the acceptability of village health workers' roles in improving maternal health care in Gombe State, Nigeria a qualitative exploration from women beneficiaries. PloS one, 15(10), e0240798.

Alderdice, F., McNeill, J., \& Lynn, F. (2013). A systematic review of systematic reviews of interventions to improve maternal mental health and well-being. Midwifery, 29(4), 389-399.

Arksey, H., \& O'Malley, L. (2005). Scoping studies: towards a methodological framework. International journal of social research methodology, 8(1), 19-32.

Armstrong, R., Hall, B. J., Doyle, J., \& Waters, E. (2011). Cochrane Update. 'Scoping the scope' of a cochrane review. J Public Health (Oxf), 33(1), 147-150. doi:10.1093/pubmed/fdr015

Balde, M. D., Bangoura, A., Sall, O., Balde, H., Niakate, A. S., Vogel, J. P., \& Bohren, M. A. (2017). A qualitative study of women's and health providers' attitudes and acceptability of mistreatment during childbirth in health facilities in Guinea. Reproductive health, 14(1), 1-13.

Bucyibaruta, B. J., Eyles, J., Harris, B., Kabera, G., Oboirien, K., \& Ngyende, B. (2018). Patients' perspectives of acceptability of ART, TB and maternal health services in a subdistrict of Johannesburg, South Africa. BMC health services research, 18(1), 1-15.

Cameron, S. T., Craig, A., Sim, J., Gallimore, A., Cowan, S., Dundas, K., . . Lakha, F. (2017). Feasibility and acceptability of introducing routine antenatal contraceptive counselling and provision of contraception after delivery: the APPLES pilot evaluation. Bjog, 124(13), 2009-2015. doi:10.1111/1471-0528.14674

Cummins, A., Griew, K., Devonport, C., Ebbett, W., Catling, C., \& Baird, K. (2021). Exploring the value and acceptability of an antenatal and postnatal midwifery continuity of care model to women and midwives, using the Quality Maternal Newborn Care Framework. Women and Birth.

Dijkers, M. (2015). What is a scoping review? KT Update, 4 (1). In.

Dillip, A., Alba, S., Mshana, C., Hetzel, M. W., Lengeler, C., Mayumana, I., . . Obrist, B. (2012). Acceptability-a neglected dimension of access to health care: findings from a study on childhood convulsions in rural Tanzania. BMC health services research, 12(1), 1-11.

Donabedian, A. (2002). An introduction to quality assurance in health care: Oxford University Press.

Dyer, T. A., Owens, J., \& Robinson, P. G. (2016). The acceptability of healthcare: from satisfaction to trust. Community Dent Health, 33(4), 242-251.

Feinberg, E., Smith, M. V., \& Naik, R. (2009). Ethnically diverse mothers' views on the acceptability of screening for maternal depressive symptoms during pediatric well-child visits. Journal of health care for the poor and underserved, 20(3), 780.

Gilson, L. (2007). Acceptability, Trust and Equity. Cambridge: University Press.

Grant, M., Wilford, A., Haskins, L., Phakathi, S., Mntambo, N., \& Horwood, C. M. (2017). Trust of community health workers influences the acceptance of community-based maternal and child health services. African Journal of Primary Health Care and Family Medicine, 9(1), 1-8.

Hadfield, H., \& Wittkowski, A. (2017). Women's Experiences of Seeking and Receiving Psychological and Psychosocial Interventions for Postpartum Depression: A Systematic Review and Thematic Synthesis of the Qualitative Literature. J Midwifery Womens Health, 62(6), 723-736. doi:10.1111/jmwh.12669

Kozarewicz, P. (2014). Regulatory perspectives on acceptability testing of dosage forms in children. International journal of pharmaceutics, 469(2), 245-248.

Kyei-Nimakoh, M., Carolan-Olah, M., \& McCann, T. V. (2017). Access barriers to obstetric care at health facilities in sub-Saharan Africa-a systematic review. Systematic reviews, 6(1), 1-16.

Liberati, A., Altman, D. G., Tetzlaff, J., Mulrow, C., Gøtzsche, P. C., loannidis, J. P., . . Moher, D. (2009). The PRISMA statement for reporting systematic reviews and meta-analyses of studies that evaluate health care interventions: explanation and elaboration. Journal of clinical epidemiology, 62(10), e1-e34. 
McIntyre, D., Thiede, M., \& Birch, S. (2009). Access as a policy-relevant concept in low- and middleincome countries. Health Econ Policy Law, 4(Pt 2), 179-193. doi:10.1017/s1744133109004836

Murphy, A. L., \& Gardner, D. M. (2019). Pharmacists' acceptability of a men's mental health promotion program using the Theoretical Framework of Acceptability. AIMS Public Health, 6(2), 195-208. doi:10.3934/publichealth.2019.2.195

Påfs, J., Musafili, A., Binder-Finnema, P., Klingberg-Allvin, M., Rulisa, S., \& Essén, B. (2015). 'They would never receive you without a husband': paradoxical barriers to antenatal care scale-up in Rwanda. Midwifery, 31(12), 1149-1156.

Penchansky, R., \& Thomas, J. W. (1981). The concept of access: definition and relationship to consumer satisfaction. Medical care, 127-140.

Rothstein, J. D., Jennings, L., Moorthy, A., Yang, F., Gee, L., Romano, K., . . . LeFevre, A. E. (2016). Qualitative assessment of the feasibility, usability, and acceptability of a mobile client data app for community-based maternal, neonatal, and child care in rural Ghana. International journal of telemedicine and applications, 2016.

Russell, D. J., Humphreys, J. S., Ward, B., Chisholm, M., Buykx, P., McGrail, M., \& Wakerman, J. (2013). Helping policy-makers address rural health access problems. Aust $J$ Rural Health, 21(2), 61-71. doi:10.1111/ajr.12023

Sekhon, M., Cartwright, M., \& Francis, J. J. (2017). Acceptability of healthcare interventions: an overview of reviews and development of a theoretical framework. BMC health services research, 17(1), 1-13.

Sekhon, M., Cartwright, M., \& Francis, J. J. (2018). Acceptability of health care interventions: A theoretical framework and proposed research agenda. In: Wiley Online Library.

Shaw, R. L., Larkin, M., \& Flowers, P. (2014). Expanding the evidence within evidence-based healthcare: thinking about the context, acceptability and feasibility of interventions. BMJ Evidence-Based Medicine, 19(6), 201-203.

Silal, S. P., Penn-Kekana, L., Harris, B., Birch, S., \& McIntyre, D. (2012). Exploring inequalities in access to and use of maternal health services in South Africa. BMC health services research, 12, 120.

Sripad, P., Warren, C. E., Hindin, M. J., \& Karra, M. (2019). Assessing the role of women's autonomy and acceptability of intimate-partner violence in maternal health-care utilization in 63 lowand middle-income countries. International journal of epidemiology, 48(5), 1580-1592.

Staneva, A. A., Bogossian, F., \& Wittkowski, A. (2015). The experience of psychological distress, depression, and anxiety during pregnancy: A meta-synthesis of qualitative research. Midwifery, 31(6), 563-573.

Staniszewska, S., Crowe, S., Badenoch, D., Edwards, C., Savage, J., \& Norman, W. (2010). The PRIME project: developing a patient evidence-base. Health Expect, 13(3), 312-322. doi:10.1111/j.1369-7625.2010.00590.x

Traylor, C. S., Johnson, J., Kimmel, M. C., \& Manuck, T. A. (2020). Effects of psychological stress on adverse pregnancy outcomes and non-pharmacologic approaches for reduction: an expert review. American Journal of Obstetrics \& Gynecology MFM, 100229.

Tricco, A. C., Lillie, E., Zarin, W., O'Brien, K. K., Colquhoun, H., Levac, D., . . Weeks, L. (2018). PRISMA extension for scoping reviews (PRISMA-SCR): checklist and explanation. Annals of internal medicine, 169(7), 467-473. 